\section{A Unique Presentation and Clinical Course of a Pancreatic Vasoactive Intestinal Polypeptide Secreting Tumor in a 68-year-old Male}

\author{
Balaji R. Jagdish, B.S. ${ }^{1}$,
}

William R. Kilgore III, M.D., FACP, AGAF 2,3

${ }^{1}$ Kansas City University of Medicine and Biosciences, Kansas City, MO

${ }^{2}$ Ascension Via Christi, Wichita, KS

${ }^{3}$ University of Kansas School of Medicine-Wichita,

Department of Internal Medicine, Wichita, KS

Received April 29, 2020; Accepted for publication. July 27, 2020; Published online Oct. 20, 2020 https://oi.org/10.17161/kijm.voll3.14763

\section{INTRODUCTION}

Chronic diarrhea, which is defined as having diarrhea lasting more than one month, affects about $3 \%-5 \%$ of the population annually. Patients often present with stooling greater than the upper normal limit of $200 \mathrm{~g} /$ day. The differential diagnosis of chronic diarrhea is extensive as there are numerous etiologies, including secretory disturbances, osmotic disturbances, malabsorption irregularities, inflammatory conditions, and issues with gut motility. Of the secretory etiologies responsible for chronic diarrhea, one such member is neuroendocrine tumors. This case report focused on the vasoactive intestinal polypeptide secreting tumors (VIPomas), a rare type of pancreatic islet tumor with an incidence of 0.05 - 0.1 per 100,000 people per year that can produce voluminous amounts of diarrhea in patients. ${ }^{2}$

\section{CASE REPORT}

A 68-year-old Caucasian male with no significant past medical history was evaluated in the emergency department for a four-month history of progressive vomiting, nausea, and diarrhea ( 15 non-bloody water-like loose stools per day) accompanied by an unintentional 35 pound weight loss. A preliminary computed-tomography (CT) report from an outside clinic reportedly had shown a mass of the pancreatic head with a duct dilation of $2.4 \mathrm{~cm}$. Upon admission, he presented with hypokalemia, hyperchloremia, hyperglycemia, and a normal lipase level. His electrolytes subsequently were replenished, and ondansetron was administered for nausea. Chromogranin A, VIP levels, stool cultures to rule out infectious etiology, CA 19-9, endoscopic ultrasound with fine needle biopsy (EUS-FNA), and fecal elastase were ordered. After two days in the hospital, his nausea/ vomiting resolved, and his diarrhea was reduced markedly. He was subsequently discharged to obtain an outpatient EUS-FNA.

Two days after discharge, the patient was re-admitted with worsening nausea, diarrhea, and vomiting. He presented with a new afebrile leukocytosis (white blood count of 19,300 per $\mu$ ), hyponatremia, hypokalemia, hyperchloremia, hyperglycemia, newly elevated liver enzymes (ALT-110 U/L, AST 49 U/L, Alkaline Phosphate 136 U/L; liver enzymes upon prior discharge were ALT 29 U/L, AST 16 U/L, Alkaline Phosphate $73 \mathrm{U} / \mathrm{L}$ ), and an elevated lipase level (53 U/L). Stool cultures with polymerase chain reaction, Chromogranin A levels, and CA 19-9 levels returned within normal range.

Due to his leukocytosis and concerns of possible infection/sepsis,

\section{KANSAS JOURNAL of MEDICINE}

ceftriaxone and metronidazole were administered. Repeat CT of the abdomen/pelvis showed mild atrophy of the pancreas with some prominence of the pancreatic duct, but was otherwise unremarkable and suggestive of gastroenteritis. Subsequent magnetic resonance cholangiopancreatography (MRCP) was unremarkable without any abnormalities of the pancreas or the pancreatic duct. Esophagogastroduodenoscopy (EGD) and colonoscopy with biopsy were conducted to evaluate for Whipple's disease, Celiac sprue, and microscopic colitis. 5-HIAA 24-hr urine levels also were ordered with concern for possible carcinoid tumor.

EGD biopsies showed moderate chronic gastritis in the antrum but were otherwise unremarkable. Colonoscopy with biopsy was unremarkable. Seven days after his first admission and one day after both the EGD/colonoscopy were performed, VIP levels returned at $296 \mathrm{pg} / \mathrm{mL}$ (normal limit $<75 \mathrm{pg} / \mathrm{mL}$ ). Subsequently, octreotide was started and EUS-FNA was performed the next day. EUS-FNA showed a $37.5 \mathrm{~mm}$ x $29.2 \mathrm{~mm}$ hypoechoic lesion in the pancreatic head and uncinate process that was obstructing the pancreatic duct. Immunohistochemistry of the EUS-FNA biopsy demonstrated positive staining for chromogranin and synaptophysin, markers confirmatory for pancreatic neuroendocrine tumor (PNT). Given that our patient's tumor was localized to the head of the pancreas (Figures 1 - 3) without any evidence of metastasis or local invasion, a Whipple procedure was performed for total tumor resection. Seven months post-Whipple procedure, single-photo emission computed tomography of the abdomen/pelvis along with $\mathrm{CT}$ of the abdomen/pelvis continued to show no evidence of metastasis.

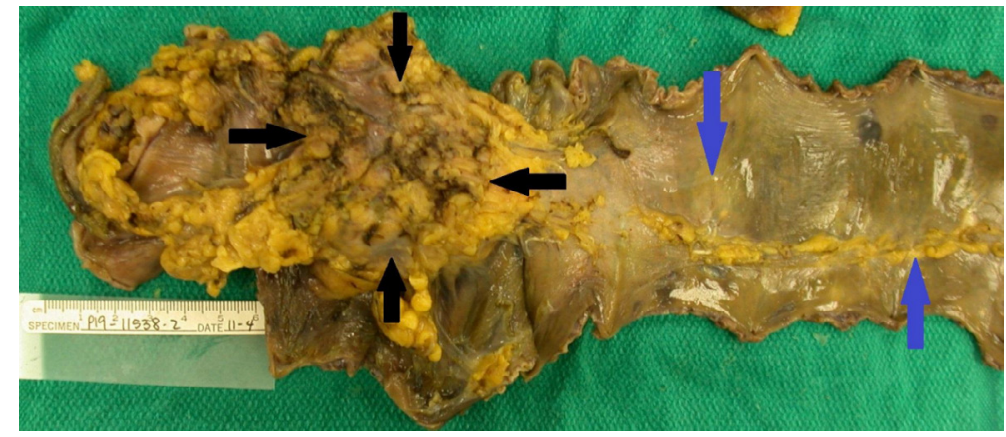

Figure l. Gross anatomy of resected specimen status post Whipple procedure. Black arrows indicate VIPoma margins. Blue arrows indicate the duodenum.

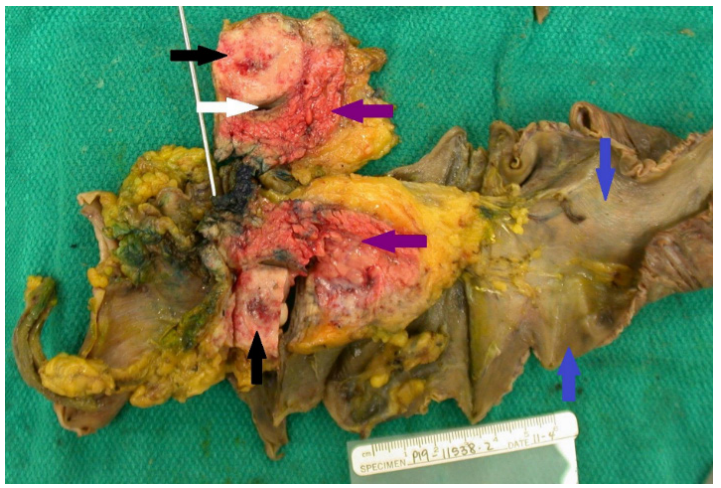

Figure 2. Gross anatomy of VIPoma bisected. Black arrow indicates VIPoma. White arrow indicates pancreatic duct. Purple arrows indicate normal pancreatic tissue. Blue arrows indicate the duodenum. 
KANSAS JOURNAL of MEDICINE 68-YEAR-OLD MALEWITHA UNIQUE VIPOMA continued.

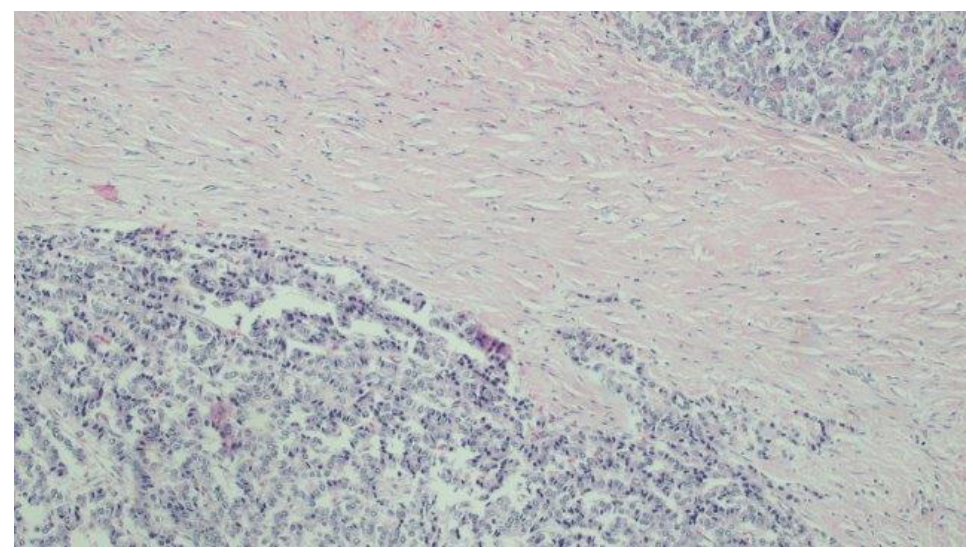

Figure 3. 10x H and E stain of VIPoma reveals tumor cells on the bottom with normal pancreatic cells on top with fibrosis (pink stained cells) between them.

\section{DISCUSSION}

VIPomas secrete VIP, a 28 amino acid polypeptide, that binds to receptors on intestinal epithelial cells and increases downstream production of cAMP via activation of cellular adenylate cyclase. ${ }^{3}$ These tumors are often present in a bimodal distribution, affecting young children and middle-aged adults based on cell origin. ${ }^{4}$ The average age of individuals affected with VIPomas of neurogenic origin is 7.3 years, while those affected with ones of pancreatic origin is 50.5 years. Pancreatic VIPomas are found more commonly in the pancreatic tail and greater likely to undergo metastasis than neurogenic VIPomas. ${ }^{4,5}$ Eighty percent of patients with pancreatic VIPomas have metastasis of the tumor at the time of diagnosis. ${ }^{5}$ Metastasis is localized most commonly to the liver and regional lymph nodes. ${ }^{6}$ This was unique to our case as our patient's VIPoma was in the head of the pancreas and had not shown to have metastasized at the time of diagnosis via CT of the abdomen, pelvis, and thorax. Additionally, despite no evidence of metastasis or obstruction of his hepatic/ common bile duct, our patient presented with elevated liver enzymes upon his re-admission. This could have been caused by dehydration and zonal necrosis secondary to the extensive diarrhea and vomiting our patient experienced while at home post-discharge.

While classically VIPomas are associated with the triad of watery diarrhea persistent with fasting, hypokalemia, and achlorhydria, patients also frequently present with dehydration, hyperglycemia, hypercalcemia, and flushing. ${ }^{5}$ The loss of potassium and bicarbonate through diarrhea can cause metabolic acidosis within patients. ${ }^{2}$ Patients often present with VIP levels $>75 \mathrm{pg} / \mathrm{dL}$, although levels $>$ $200 \mathrm{pg} / \mathrm{dL}$ can be considered diagnostic. ${ }^{5}$ While our patient presented with electrolyte imbalances, such as hyperglycemia, hypokalemia, and hyperchloremic metabolic acidosis, he additionally presented with hyponatremia and watery diarrhea that moderately improved with fasting. He also presented with an unexplained afebrile leukocytosis on re-admission, a finding that originally made the medical team consider a potential infectious etiology.

Imaging is useful in the diagnosis and localization of VIPomas. While ultrasound can be used for initial imaging, contrast CT and magnetic resonance imaging (MRI) confer greater detail in localizing the tumors. Triple-phase CT scan is often the modality of choice for initial diagnosis; however, MRI is more sensitive and specific for locating the primary pancreatic VIPoma and any liver metastasis that might be present. ${ }^{4}$ However, CT and MRI localize less than $10 \%$ of PNTs less than $1 \mathrm{~cm}$ in diameter and only $30 \%-40 \%$ of those measuring $1-3 \mathrm{~cm}$ in diameter. ${ }^{7}$ EUS-FNA also has been shown to be effective in locating PNTs, with one study finding the diagnostic sensitivity of EUS-FNA in PNTs to be $87 \% .^{7}$ Newer effective diagnostic modalities include somatostatin receptor positivity and gallium-labeled somatostatin analogs with positron emission tomography/CT.,7

Our case report suggested supporting the notion that EUS-FNA might be a superior tool than MRI and CT imaging in diagnosing VIPomas. While the original CT from an outside clinic indicated a mass of the pancreatic head, repeat CT of the abdomen along with MRCP at our institution had failed to indicate any presence of such a mass. This led our team to consider other etiologies of secretory diarrhea, such as Whipple's disease and microscopic colitis, followed by appropriate workup. It was not until the EUS-FNA that his tumor was discovered.

Initial treatment consists of electrolyte correction, specifically hypokalemia, along with administration of somatostatin analogs such as octreotide. ${ }^{2-4,8}$ Next, options such as surgery, chemotherapy, and radiotherapy should be considered based on the location and extent of metastasis of the VIPoma. ${ }^{3}$ As previously stated, our patient underwent a Whipple procedure for total tumor resection.

Overall, while our patient presented with some classical symptoms of a VIPoma, his unique presentation influenced our clinical course. His case not only demonstrated the efficacy of EUS-FNA in diagnosing VIPomas and the variability surrounding these tumors in terms of presentation, but also the need for continued research to understand further the complexity behind VIPomas.

\section{REFERENCES}

${ }^{1}$ Schiller LR, Pardi DS, Spiller R, et al. Gastro 2013 APDW/WCOG Shanghai Working Party Report: Chronic diarrhea: Definition, classification, diagnosis. J Gastroenterol Hepatol 2014; 29(1):6-25. PMID: 24117999.

${ }^{2}$ Dimitriadis GK, Weickert MO, Randeva HS, Kaltsas G, Grossman A. Medical management of secretory syndromes related to gastroenteropancreatic neuroendocrine tumours. Endocr Relat Cancer 2016; 23(9):R423-436. PMID: 27461388.

Belei OA, Heredea ER, Boeriu E, et al. Verner-Morrison syndrome. Literature review. Rom J Morphol Embryol 2017; 58(2):371-376. PMID: 28730220.

${ }^{4}$ Siddappa PK, Vege SS. Vasoactive intestinal peptide-secreting tumors: A review. Pancreas 2019; 48(9):1119-1125. PMID: 31609932.

5 Parbhu SK, Adler DG. Pancreatic neuroendocrine tumors: Contemporary diagnosis and management. Hosp Pract 2016; 44(3):109-119. PMID: 27404266.

${ }^{6}$ Smith SL, Branton SA, Avino AJ, et al. Vasoactive intestinal polypeptide secreting islet cell tumors: A 15-year experience and review of the literature. Surgery 1998; 124(6):1050-1055. PMID: 9854582.

7 Pais SA, Al-Haddad M, Mohamadnejad M, et al. EUS for pancreatic neuroendocrine tumors: A single-center, 11-year experience. Gastrointest Endos 2010; 71(7):1185-1193. PMID: 20304401.

8 Perry RR, VinikAI. Clinical review 72: Diagnosis and management of functioning islet cell tumors. J Clin Endocrinol Metab 1995; 80(8):2273-2278. PMID: 7629220.

Keywords: VIPomas, pancreatic neoplasm, neuroendocrine tumors, diarrhea 